\title{
Sensitisation of colonic nociceptors by TNFa is dependent on TNFR1 expression and p38 MAPK activity
}

\author{
Katie H. Barker ${ }^{1}$, James P. Higham ${ }^{1}$, Luke A. Pattison ${ }^{1}$, Toni S. Taylor ${ }^{1}$, lain P. Chessell, \\ Fraser Welsh², Ewan St. J. Smith', David C. Bulmer'. \\ ${ }^{1}$ Department of Pharmacology, University of Cambridge, Cambridge CB2 1PD, UK \\ ${ }^{2}$ Neuroscience, BioPharmaceuticals R\&D, AstraZeneca, Cambridge.
}

Key words: Visceral pain; Tumour Necrosis Factor-alpha, TRPV1, capsaicin, p38 MAPK, Inflammatory bowel disease, Irritable bowel syndrome, nociception

Number of pages: 31

Number of figures and tables: 9

Corresponding Author

Dr David Bulmer:

Department of Pharmacology, University of Cambridge,

Tennis Court Road, Cambridge CB2 1PD

Email: dcb53@cam.ac.uk

Tel: $+44(0) 1223334000$

Fax: $+44(0) 1223334100$

https://www.phar.cam.ac.uk/research/bulmer 


\begin{abstract}
Visceral pain is a leading cause of morbidity in gastrointestinal diseases, which is exacerbated by the gut related side-effects of many analgesics. New treatments are needed and further understanding of the mediators and mechanisms underpinning visceral nociception in disease states is required to facilitate this. The pro-inflammatory cytokine TNFa is linked to pain in patients with inflammatory bowel disease or irritable bowel syndrome and has been shown to sensitise colonic sensory neurons. Somatic, TNFatriggered thermal and mechanical hypersensitivity is mediated by TRPV1 signalling and p38 MAPK activity respectively, downstream of TNFR1 receptor activation. We therefore hypothesised that TNFR1-evoked p38 MAPK activity may also be responsible for TNFa sensitisation of colonic afferent responses to the TRPV1 agonist capsaicin, and noxious distension of the bowel. Using $\mathrm{Ca}^{2+}$ imaging of dorsal root ganglia sensory neurons, we observed TNFa-mediated increases in intracellular $\left[\mathrm{Ca}^{2+}\right]$ and sensitisation of capsaicin responses. The sensitising effects of TNFa were dependent on TNFR1 expression and attenuated by TRPV1 and p38 MAPK inhibition. Consistent with these findings, ex vivo colonic afferent fibre recordings demonstrated enhanced response to noxious ramp distention of the bowel and bath application of capsaicin following TNFa pre-treatment. Responses were reversed by p38 MAPK inhibition and absent in tissue from TNFR1 knockout mice. Our findings demonstrate a contribution of TNFR1, p38 MAPK and TRPV1 to TNFa-induced sensitisation of colonic afferents, highlighting the potential utility of these drug targets for the treatment of visceral pain in Gl disease.
\end{abstract}




\section{Key Points Summary}

- TNFa sensitises sensory neurons and colonic afferents to the TRPV1 agonist capsaicin.

- TNFa mediated sensitisation of sensory neurons and colonic nociceptors is dependent on TNFR1 expression.

- TNFa sensitisation of sensory neurons and colonic afferents to capsaicin and noxious ramp distension is abolished by inhibition of p38 MAPK. 


\section{Introduction}

Abdominal pain is a leading cause of morbidity in gastrointestinal (GI) diseases, such as inflammatory bowel disease (IBD), and irritable bowel syndrome (IBS) (Grundy et al., 2019; Spiller \& Major, 2016). Mechanistically, the sensitisation of visceral nociceptors by inflammation, infection, allergy, and other pathological processes, is a principal cause of pain in disease states, although the mediators and mechanisms underpinning this are not yet fully understood (Grundy et al., 2019; McMahon et al., 2015). The proinflammatory cytokine tumour necrosis factor a (TNFa) has been implicated in the development of peripheral sensitisation and visceral pain in IBD and IBS patients, based on its causative role in inflammatory disease pathology, localised release from mast cells, and the significant correlation between pain scores and peripheral blood mononuclear cell (PBMC)-evoked TNFa release in IBS patients (Hughes et al., 2013; Rijnierse et al., 2006). These observations are supported by expression of the TNFR1 receptor in mouse colonic sensory neurons (Hockley et al., 2019) and human dorsal root ganglia (DRG) (Wangzhou et al., 2020), as well as functional data demonstrating the sensitisation of colonic neurons and colonic afferents by TNFa (Hughes et al., 2013). These effects have been linked to enhanced tetrodotoxin (TTX) -resistant voltage gated sodium channel currents ( $\mathrm{Na}_{v} 1.8$ subtype) (Jin \& Gereau IV, 2006) and suppressed voltage gated potassium channel currents (delayed rectifier subtypes), downstream of TNFR1 receptor activation in DRG neurons (Ibeakanma \& Vanner, 2010), and TRPA1 channel activity in colonic afferents (Hughes et al., 2013). The translational importance of these findings is supported by studies utilising IBD patient colonic biopsy supernatants or IBS patient PBMCs, which have confirmed the essential contribution of TNFa to the respective sensitisation of DRG neurons or colonic afferents using tissue from TNFR $1^{-/}$mice or pre-treatment with the anti-TNFa monoclonal antibody infliximab (Hughes et al., 2013; Ibeakanma \& Vanner, 2010).

In addition to visceral pain, TNFa also evokes somatic, thermal, and mechanical hypersensitivity by increasing TRPV1 activity (Khan et al., 2008) and p38 mitogen-activated protein kinase (MAPK)-mediated Nav1.8 activity, downstream of TNFR1 receptor activation (Jin \& Gereau IV, 2006). Given TRPV1 and Nav1.8 channels are co-expressed with TNFR1 in colonic nociceptors (Hockley et al., 2019), we reasoned that TNFa may sensitise responses to the TRPV1 agonist capsaicin, and that p38 MAPK signalling may be responsible for TNFa-mediated sensitisation of colonic nociceptors. Consequently, the aims of this study were to investigate the contribution of p38 MAPK and TNFR1 to TNFamediated sensitisation of colonic afferents to capsaicin and noxious distension of the bowel using a combination of $\mathrm{Ca}^{2+}$ imaging of DRG neurons and ex vivo electrophysiological recordings of colonic afferent activity. 


\section{Materials and Methods}

\section{Ethical Approval}

All animal experiments were conducted in compliance with the Animals (Scientific Procedures) Act 1986 Amendment Regulations 2012 under Project Licence P7EBFC1B1 granted to E. St. J. Smith by the Home Office and approval by the University of Cambridge Animal Welfare Ethical Review Body.

\section{Reagents}

Stock concentrations of TNFa $\left(0.1 \mathrm{mg} / \mathrm{ml} ; \mathrm{H}_{2} \mathrm{O}\right.$ with $0.2 \%(\mathrm{w} / \mathrm{v})$ bovine serum albumin), capsaicin (1 mM; 100\% ethanol) and staurosporine (10 mM; DMSO) were dissolved as described, all purchased from Sigma-Aldrich. R7050 (10 mM; DMSO), thapsigargin (1 mM; DMSO), A425619 (1 mM; DMSO) and SB203580 (10 mM; DMSO) were obtained from Tocris and stock concentrations made up as described. Nifedipine (100 mM; DMSO) and atropine (100 mM; 100\% ethanol) were purchased from Sigma-Aldrich and dissolved as described. All drugs were diluted to working concentrations in extracellular solution (ECS) or Krebs buffer on the day of the experiment.

\section{Animals}

Adult male C57BL/6J mice (8-16 weeks) were obtained from Charles River (Cambs, UK; RRID:IMSR_JAX:000664). Mice were conventionally housed in temperature-controlled rooms $\left(21^{\circ} \mathrm{C}\right)$ with a $12-\mathrm{h}$ light/dark cycle and provided with nesting material, a red plastic shelter and access to food and water ad libitum. TNFR $1^{-1}$ mice (9-14 weeks; 8F, 7M; Jackson Laboratory, ME, USA; RRID:IMSR_JAX:003242) were housed in individually ventilated plastic cages under the same conditions.

\section{Primary culture of mouse dorsal root ganglia neurons}

DRG neurons were cultured as previously described (Hockley et al., 2019). Briefly, mice were euthanised by exposure to a rising concentration of $\mathrm{CO}_{2}$, followed by cervical dislocation. Isolated DRG (T12-L5, spinal segments innervating the distal colon) were incubated in $1 \mathrm{mg} / \mathrm{ml}$ collagenase $(15 \mathrm{~min})$ followed by trypsin $(1 \mathrm{mg} / \mathrm{ml})$ both with $6 \mathrm{mg} / \mathrm{ml}$ BSA in Leibovitz's L-15 Medium, GlutaMAX'M Supplement (supplemented with $2.6 \%(\mathrm{v} / \mathrm{v})$ $\mathrm{NaHCO}_{3}$ ). DRG were resuspended in $2 \mathrm{ml}$ Leibovitz's L-15 Medium, GlutaMAXTM Supplement containing $10 \%(\mathrm{v} / \mathrm{v})$ foetal bovine serum (FBS), $2.6 \%(\mathrm{v} / \mathrm{v}) \mathrm{NaHCO}_{3}, 1.5 \%(\mathrm{v} / \mathrm{v})$ glucose and 300 units $/ \mathrm{ml}$ penicillin and $0.3 \mathrm{mg} / \mathrm{ml}$ streptomycin (P/S). DRG were mechanically dissociated, centrifuged $(1000 \mathrm{rpm})$ and the supernatant collected for five triturations. Following centrifugation and resuspension, the supernatant $(50 \mu \mathrm{L})$ was plated 
onto $35 \mathrm{~mm}$ poly-D-lysine coated glass bottom culture dishes (MatTek, MA, USA), and further coated with laminin (Thermo Fisher: 23017015). Dishes were incubated for 3 hours to allow cell adhesion, before flooding with $2 \mathrm{ml}$ Leibovitz's L-15 Medium, GlutaMAX'M Supplement containing $10 \%$ (v/v) FBS, $2.6 \%(\mathrm{v} / \mathrm{v}) \mathrm{NaHCO}_{3}, 1.5 \%(\mathrm{v} / \mathrm{v})$ glucose and $\mathrm{P} / \mathrm{S}$ and cultured for 24 hours. All incubations were carried out at $37^{\circ} \mathrm{C}$ with $5 \% \mathrm{CO}_{2}$.

\section{$\mathrm{Ca}^{2+}$ imaging}

Extracellular solution (in mM: $140 \mathrm{NaCl}, 4 \mathrm{KCl}, 1 \mathrm{MgCl}_{2}, 2 \mathrm{CaCl}_{2}, 4$ glucose, $10 \mathrm{HEPES}$ ) was prepared and adjusted to $\mathrm{pH} 7.4$ using $\mathrm{NaOH}$ and an osmolality of 290-310 mOsm using sucrose. Cells were incubated for $30 \mathrm{~min}$ with $100 \mu \mathrm{l}$ of $10 \mu \mathrm{M} \mathrm{Ca}^{2+}$ indicator Fluo-4-AM (room temperature; shielded from light). For inhibitor studies requiring pre-incubation, $200 \mu \mathrm{l}$ of drug was added for 10 min prior to imaging.

Dishes were mounted on the stage of an inverted microscope (Nikon Eclipse TE-2000S) and cells were visualised at $10 \times$ magnification with brightfield illumination. Cells were initially superfused with ECS, or drug in inhibitor studies to establish baseline. For studies in which $\mathrm{Ca}^{2+}$ was absent from ECS, bath solution was made up as follows (in $\mathrm{mM}$ ): $140 \mathrm{NaCl}, 4 \mathrm{KCl}$, $2 \mathrm{MgCl}_{2}$, 4 glucose, 10 HEPES, 1 EGTA (pH 7.35-7.45 with $\mathrm{NaOH}$; 290-310 mOsm with sucrose). To compensate for the loss of extracellular divalent cations, the $\mathrm{MgCl}_{2}$ concentration was increased and EGTA was used to chelate any remaining $\mathrm{Ca}^{2+}$.

Fluorescent images were captured with a CCD camera (Rolera Thunder, Qimaging, MC, Canada or Retiga Electro, Photometrics, AZ, USA) at $2.5 \mathrm{fps}$ with $100 \mathrm{~ms}$ exposure and a $470 \mathrm{~nm}$ light source for excitation of Fluo-4-AM (Cairn Research, Faversham, UK). Emission at $520 \mathrm{~nm}$ was recorded with $\mu$ Manager (Edelstein et al., 2014). All protocols began with a $10 \mathrm{~s}$ baseline of ECS before drug superfusion. With multiple drug additions to the same dish, cells were allowed 4 min recovery between applications. Finally, cells were stimulated with $50 \mathrm{mM} \mathrm{KCl}$ for $10 \mathrm{~s}$ to determine cell viability, identify neuronal cells and allow normalisation of fluorescence. A fresh dish was used for each protocol and all solutions were diluted in ECS.

\section{$\mathrm{Ca}^{2+}$ imaging data analysis}

Individual cells were circled on a brightfield image and outlines overlaid onto fluorescent images using ImageJ ( $\mathrm{NIH}, \mathrm{MA}$, USA). Pixel intensity was measured and analysed with custom-written scripts in RStudio (RStudio, MA, USA). Background fluorescence was subtracted from each cell, and fluorescence intensity (F) baseline corrected and normalised to the maximum fluorescence elicited during $50 \mathrm{mM} \mathrm{KCl}$ stimulation ( $\left.F_{\text {pos }}\right)$. Maximum $\mathrm{KCl}$ fluorescence was denoted as $1 \mathrm{~F} / \mathrm{F}_{\text {pos. }}$. Further analysis was confined to cells with a 
fluorescence increase $\geq 5$ standard deviations above the mean baseline before $50 \mathrm{mM} \mathrm{KCl}$ application. Neurons were deemed responsive to a drug challenge if a fluorescence increase of $0.1 \mathrm{~F} / \mathrm{F}_{\text {pos }}$ was seen in response to drug perfusion. The proportion of responsive neurons and magnitude of the fluorescence response was measured for each experiment, with peak responses calculated from averaging fluorescence values of individual neurons at each time point.

\section{Magnetic-activated cell sorting}

To determine the role of satellite cells, such as glia, in the neuronal responses to TNFa, satellite cells were removed from DRG cultures using magnetic-activated cell sorting (MACS), with equipment purchased from Miltenyi Biotec and using protocols previously described (Thakur et al., 2014).

DRG from 2-3 mice were isolated and cultured as above, but trypsin incubation was omitted and DRG were incubated with collagenase ( $1 \mathrm{mg} / \mathrm{ml}$ with $6 \mathrm{mg} / \mathrm{ml} \mathrm{BSA})$ for $45 \mathrm{~min}$. Pelleted neurons were washed in $2 \mathrm{ml}$ Dulbecco's phosphate-buffered saline (DPBS, containing 0.9 $\mathrm{mM} \mathrm{CaCl} 2$ and $0.5 \mathrm{mM} \mathrm{MgCl}_{2}$ ) and centrifuged for $7 \mathrm{~min}$ (1000 rpm). The pellet was resuspended in MACS rinsing solution $(120 \mu \mathrm{l}$ ), supplemented with $0.5 \% \mathrm{w} / \mathrm{v}$ BSA (sterile filtered at $0.2 \mu \mathrm{M})$, and incubated $\left(5 \mathrm{~min}\right.$ at $\left.4^{\circ} \mathrm{C}\right)$ with a biotin-conjugated non-neuronal antibody cocktail $(30 \mu \mathrm{l})$. DPBS was added to a volume of $2 \mathrm{ml}$ and the suspension centrifuged for $7 \mathrm{~min}$ at $1000 \mathrm{rpm}$. The pellet was resuspended in $120 \mu \mathrm{l}$ MACS rinsing solution with $30 \mu \mathrm{L}$ biotin-binding magnetic beads and incubated for a further 10 min at $4{ }^{\circ} \mathrm{C}$, before being topped-up to $500 \mu \mathrm{l}$ with MACS buffer.

The cell suspension was filtered by gravity through a magnetic column (LD column), primed with $2.5 \mathrm{ml}$ MACS rinsing solution. Following the addition of the cell suspension, $1 \mathrm{ml}$ MACS rinsing solution was used to collect the remnants of the cell suspension and passed through the column prior to a final wash. The $5 \mathrm{ml}$ elute was centrifuged for $7 \mathrm{~min}$ at $1000 \mathrm{rpm}$ and the final pellet resuspended in supplemented L-15 medium, before plating on $35 \mathrm{~mm}$ poly-Dlysine coated glass bottom culture dishes further coated with Matrigel (diluted 1:10 in L-15 medium). Dishes were incubated for 3 hours to allow cell adhesion, after which $2 \mathrm{ml}$ Leibovitz's L-15 Medium, GlutaMAXTM Supplement containing 10\% (v/v) FBS, 2.6\% (v/v) $\mathrm{NaHCO}_{3}, 1.5 \%(\mathrm{v} / \mathrm{v})$ glucose and P/S was added, and dishes were cultured for 48 hours $\left(37^{\circ} \mathrm{C}, 5 \% \mathrm{CO}_{2}\right)$. Medium was changed after 24 hours.

\section{Immunocytochemistry of cultured DRG}

DRG neurons were cultured as above and seeded onto $12 \mathrm{~mm}$ coverslips coated in poly-Llysine and laminin. After 24-48 hours in culture, cells were fixed at room temperature in $4 \%$, 
pH 7.0 paraformaldehyde (10 $\mathrm{min})$ and washed in PBS. Cells were permeabilized with $0.05 \%$ Triton-X100 for 5 min at room temperature. Cells were washed again in PBS and then blocking buffer ( $1 \%$ goat serum in $0.2 \%$ Triton-X100) was applied. After 2 hours, cells were incubated with a rabbit anti- $\beta$ III tubulin primary antibody (1:1000, Abcam: ab18207; RRID:AB_444319) (Prado et al., 2021) for 3 hours at room temperature.

Following primary antibody incubation, cells were washed in PBS and incubated with an Alexa Fluor-568 goat anti-rabbit secondary antibody diluted in PBS (1:1000, Invitrogen: A11008; RRID:AB_143165) (Crerar et al., 2019) plus 4'-6-diamidino-2-phenylindole (DAPI; 1:1000, Abcam) for 1 hour at room temperature. After a final wash, coverslips were mounted, cell side down, on $25 \times 75 \times 1 \mathrm{~mm}$ glass slides using Mowoil 4-88 mounting medium (Sigma-Aldrich: 81381). Mounting medium was set at $4^{\circ} \mathrm{C}$ and slides were imaged within 1 hour.

Slides were imaged using an Olympus BX51 microscope. Fluorophores were excited with $568 \mathrm{~nm}$ (Alexa Fluor-568) or $350 \mathrm{~nm}$ (DAPI) light sources. Images were captured on a Qicam CCD camera (QImaging) with a $100 \mathrm{~ms}$ exposure and false coloured ( $\beta$ III tubulin, green; DAPI, blue). No $\beta$ III tubulin staining was observed when the primary antibody was omitted (data not shown).

\section{Image Analysis}

Images were analysed using ImageJ. An automatic 'minimum error' threshold algorithm was applied to 8-bit images of $\beta$ III tubulin or DAPI staining. Binary and raw images were manually compared, and the threshold manually adjusted to ensure all regions of interest were captured. Generally, the threshold was placed at the first minimum after the major peak of the image histogram. Binary images then underwent watershed segmentation to separate distinct objects in proximity. Identified particles were automatically counted to calculate the ratio of $\beta$ III tubulin-positive cells (neurons) to DAPI-positive cells (neurons and satellite cells).

\section{Ex vivo electrophysiology recordings of colonic afferent activity}

The distal colorectum and associated lumbar splanchnic nerve (LSN; rostral to inferior mesenteric ganglia) were isolated from mice euthanised as described above and cannulated in a rectangular recording chamber with Sylgard base (Dow Corning, UK). Colons were luminally perfused $(200 \mu \mathrm{l} / \mathrm{min})$ and serosally superfused $\left(7 \mathrm{ml} / \mathrm{min} ; 32-34{ }^{\circ} \mathrm{C}\right)$ with carboxygenated Krebs buffer solution (in mM: $124 \mathrm{NaCl}, 4.8 \mathrm{KCl}, 1.3 \mathrm{NaH}_{2} \mathrm{PO}_{4} \cdot \mathrm{H}_{2} \mathrm{O}, 2.5$ $\mathrm{CaCl}_{2} \cdot 2 \mathrm{H}_{2} \mathrm{O}, 1.2 \mathrm{MgSO}_{4} \cdot 7 \mathrm{H}_{2} \mathrm{O}, 11.1 \mathrm{D}$-(+)-glucose, and $25 \mathrm{NaHCO}_{3}$ ) supplemented with 10 $\mu \mathrm{M}$ atropine and $10 \mu \mathrm{M}$ nifedipine to paralyse smooth muscle activity (Ness \& Gebhart, 1988a). 
Multi-unit activity from LSN bundles were recorded using borosilicate glass suction electrodes, and signals were amplified, band pass filtered (gain $5 \mathrm{KHz} ; 100-1300 \mathrm{~Hz}$; Neurolog, Digitimer Ltd, UK), and filtered digitally for $50 \mathrm{~Hz}$ noise (Humbug, Quest Scientific, Canada). Analogue signals were digitized at $20 \mathrm{kHz}$ (Micro1401; Cambridge Electronic Design, UK). All signals were visualised using Spike2 software.

\section{Electrophysiology Protocols}

Following a minimum 30 min stabilisation period, repeated ramp distensions $(0-80 \mathrm{mmHg})$ of the colorectum were performed by occluding luminal perfusion out-flow of the cannulated tissue (total distension time taking approximately $220 \mathrm{~s}$ ). Ramp distensions at pressures > $30 \mathrm{mmHg}$ are noxious evoking pain behaviours in mice and humans (Hughes et al., 2009; Ness \& Gebhart, 1988b).

In total, five ramp distensions, were performed separated by $15 \mathrm{~min}$, after which $1 \mu \mathrm{M}$ capsaicin $(20 \mathrm{ml}$ ) was applied by bath superfusion (15 min after the last distension). TNFa (100 nM) or vehicle (buffer) was applied by luminal perfusion TNFa (15 min) was applied between the end of ramps 3 and 4 . For experiments examining the effect of p38 MAPK inhibition, preparations were pre-treated by luminal perfusion with either SB203580 (10 $\mu \mathrm{M})$ or vehicle $(0.01 \%$ DMSO) started 15 min prior to and continued throughout TNFa luminal perfusion.

\section{Electrophysiological Data Analysis}

In electrophysiological recordings, nerve discharge was determined by measuring the number of spikes passing a manually determined threshold twice the level of background noise (typically $60-80 \mu \mathrm{V}$ ) and binned to determine average firing frequency every $10 \mathrm{~s}$. Changes in neuronal firing rates were calculated by subtracting baseline firing (averaged 3 min prior to distension or drug perfusion) from increases in nerve activity following ramp distension or capsaicin application. Peak firing to noxious mechanical distension and capsaicin application was determined respectively as the highest neuronal activity during ramp distension 5 and during the $10 \mathrm{~min}$ post capsaicin application. Changes to neuronal activity were recorded with each $5 \mathrm{mmHg}$ increase in pressure and used to visualise ramp profiles. Capsaicin response profiles were plotted from binned data at $30 \mathrm{~s}$ increments. The area under the curve (AUC) was calculated for the duration of each ramp distension (0-80 $\mathrm{mmHg}$ ) and for the $10 \mathrm{~min}$ following initial capsaicin application from response profiles using GraphPad Prism 9 software.

\section{Statistical analysis}


Data sets were analysed using the appropriate statistical tests; unpaired t-tests and one-way ANOVA with Holm-Šídák's multiple comparisons test were used for $\mathrm{Ca}^{2+}$ imaging analysis of responder proportions and magnitude respectively. In electrophysiology protocols, changes in peak firing frequency were compared using unpaired t-tests and one-way ANOVA with Holm-Šídák's multiple comparisons test as required. Comparison of ramp 3 and ramp 5 AUC was analysed using a two-way ANOVA with Holm-Šídák's multiple comparisons test. Differences in ramp distension profiles were analysed with multiple unpaired t-tests to account for the change in pressure. Capsaicin response profiles were analysed using twoway ANOVA with Holm-Šídák's multiple comparisons test. All data displayed as means \pm standard deviation (SD) unless otherwise stated. 


\section{Results}

\section{TNFa sensitises TRPV1 signalling in DRG neurons}

In keeping with studies showing TNFa potentiation of TRPV1-mediated currents and $\mathrm{Ca}^{2+}$ flux in sensory neurons (Hsu et al., 2017; Khan et al., 2008), we examined the effect of overnight (24 hours) incubation, or acute application of TNFa $(3 n M)$, on capsaicin $(1 \mu \mathrm{M})$ evoked increases in intracellular $\mathrm{Ca}^{2+}$ concentration $\left[\mathrm{Ca}^{2+}\right]_{i}$ within DRG sensory neurons. Overnight incubation with TNFa elicited a significantly greater peak increase in $\left[\mathrm{Ca}^{2+}\right]_{i}$ to $10 \mathrm{~s}$ capsaicin ( $p=0.0417$, unpaired t-test; $n=5, N=5$; Figure $1 A-C$ ) in a comparable proportion of neurons compared with vehicle incubation ( $p=0.4486$, unpaired t-test; $n=5, N=5$; Figure 1D). Additionally, acute administration of TNFa (1 min), between repeat applications of capsaicin, prevented the marked desensitisation of response to the second capsaicin application (Figure 1E). This effect was only observed in a subset of capsaicin responsive neurons $\left(37.7 \pm 14.2 \%\right.$ ) that were co-sensitive to TNFa (e.g. $\operatorname{cap}_{2} / \mathrm{cap}_{1} \mathrm{p}=0.00130$, one-way ANOVA with Holm-Šídák's multiple comparisons test; $\mathrm{n}=5-6, \mathrm{~N}=5$; Figure $1 \mathrm{~F}$ ). 
A
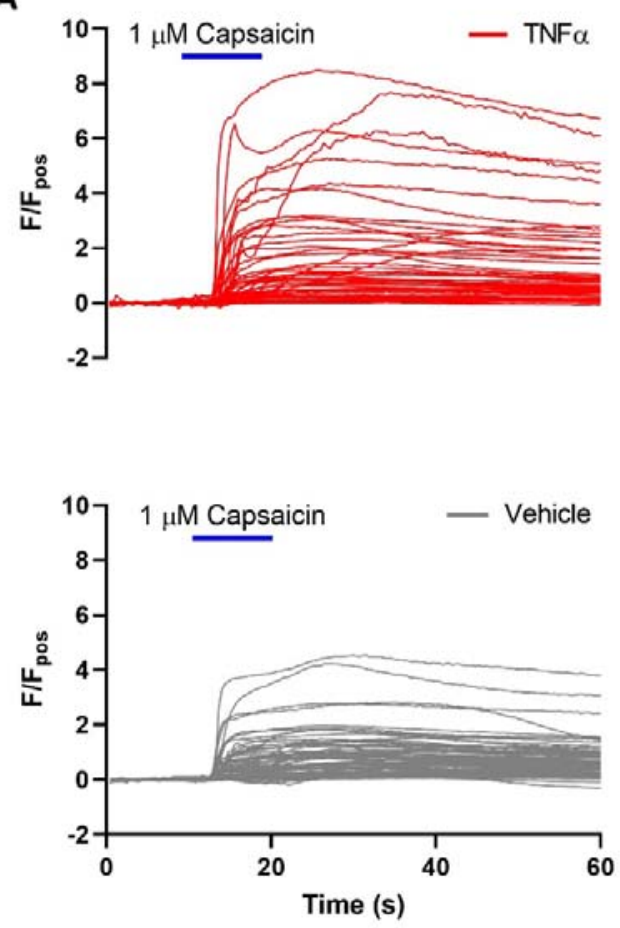

$\mathbf{E}$

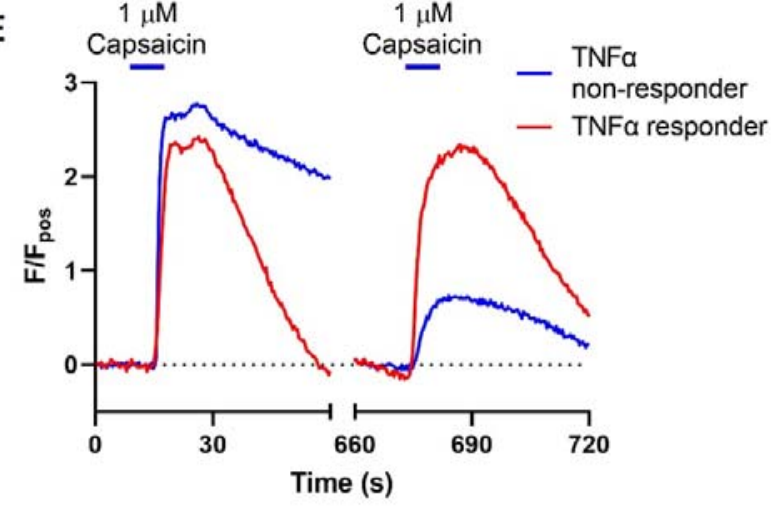

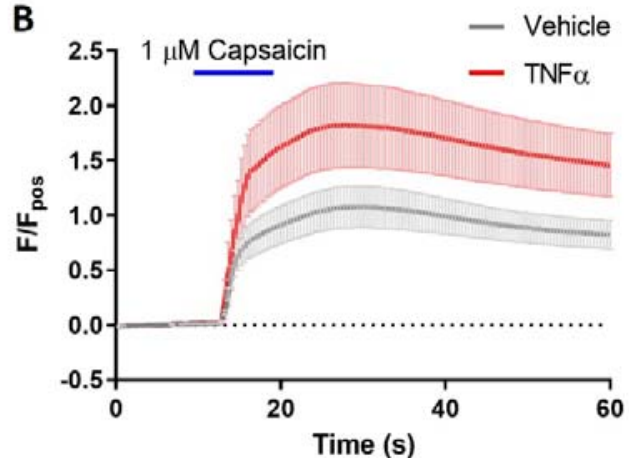
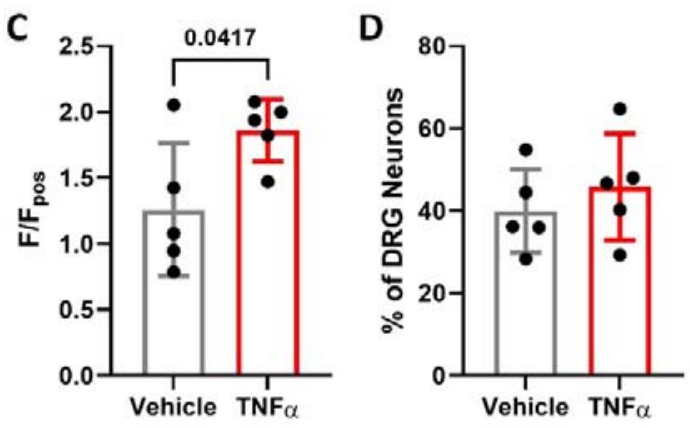

$\mathbf{F}$

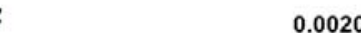

Figure 1 - TNFa sensitised capsaicin-evoked $\left[\mathrm{Ca}^{2+}\right]_{i}$ increase in DRG neurons. Representative response profiles illustrating $(A)$ the greater effect of $1 \mu \mathrm{M}$ capsaicin (10 s) on DRG neurons pre-incubated with TNFa (3 nM, top panel) compared with vehicle (PBS, bottom panel) for 24 hours; and (B) the increased averaged response of individual capsaicin sensitive neurons within a culture dish (mean \pm SEM) pre-incubated with TNFa $(n=56)$ compared with vehicle $(n=79)$. These responses resulted in $(C)$ a significantly greater peak response averaged per dish in capsaicin-sensitive DRG neurons pre-incubated with TNFa compared with vehicle ( $p=0.0417$, unpaired $t$-test; $n=5$ dishes from $N=5$ mice per group). (D) The overall proportion of capsaicin-sensitive DRG neurons was comparable between culture dishes pre-incubated with vehicle or TNFa respectively ( $p=0.4486$, unpaired t-test; $n$ 
$=5, N=5)$. In addition, the marked desensitisation of responses to a repeat capsaicin application was greatly attenuated by $T N F \alpha$, as illustrated (E) by the desensitising response profile to repeat capsaicin application in a TNFa-insensitive (blue) DRG neuron in comparison to the lack of desensitisation to capsaicin in a TNFa-sensitive (red) neuron; and confirmed in $(F)$ by the significantly greater peak response ratios between the second $\left(c_{2} p_{2}\right)$ and first ( cap $_{1}$ ) capsaicin application in DRG neurons co-sensitive to TNFa (3 nM) compared with vehicle (ECS) or TNFa insensitive neurons ( $p=0.0013$, one-way ANOVA with HolmŠídák's multiple comparisons test; $n=5-6$ dishes from $N=5-6$ independent cultures).

\section{TNFR1, TRPV1 and p38 MAPK signalling mediates the sensitisation of DRG neurons by TNFa}

Having confirmed that TNFa sensitises capsaicin signalling in DRG neurons, we next investigated the rise in $\left[\mathrm{Ca}^{2+}\right]_{i}$ elicited by $1 \mathrm{~min}$ TNFa alone. Application of TNFa elicited a concentration dependent increase in both the magnitude of response $(p=0.0415)$, and the proportion of neurons activated by TNFa ( $p=0.00290$, one-way ANOVA with Holm-Šídák's multiple comparisons test; $n=6, N=5$; Figure $2 A \& B$ ). At the highest concentration tested, $45.8 \pm 9.37 \%$ of DRG neurons were activated by TNFa, of which $79.9 \pm 10.7 \%$ were also cosensitive for capsaicin, indicating a preferential activation of nociceptors by TNFa. In DRG neurons isolated from TNFR $1^{--}$mice, the change in $\left[\mathrm{Ca}^{2+}\right]_{i}$ in response to TNFa was no different to that produced by ECS (indicated by dashed line in Figure 2C; $p=0.0001$, oneway ANOVA with Holm-Šídák's multiple comparisons test; $n=5, N=5$ ). Pre-treatment with R7050, an inhibitor of TNFR1 signalling, attenuated the TNFa-mediated rise in $\left[\mathrm{Ca}^{2+}\right]_{i}$ in DRG neurons isolated from wild type mice ( $p=0.0045$, one-way ANOVA with Holm-Šídák's multiple comparisons test; $n=5, N=5$; Figure $2 C$ ). These observations confirm an essential role for TNFR1 in TNFa-mediated nociceptor stimulation. 
A
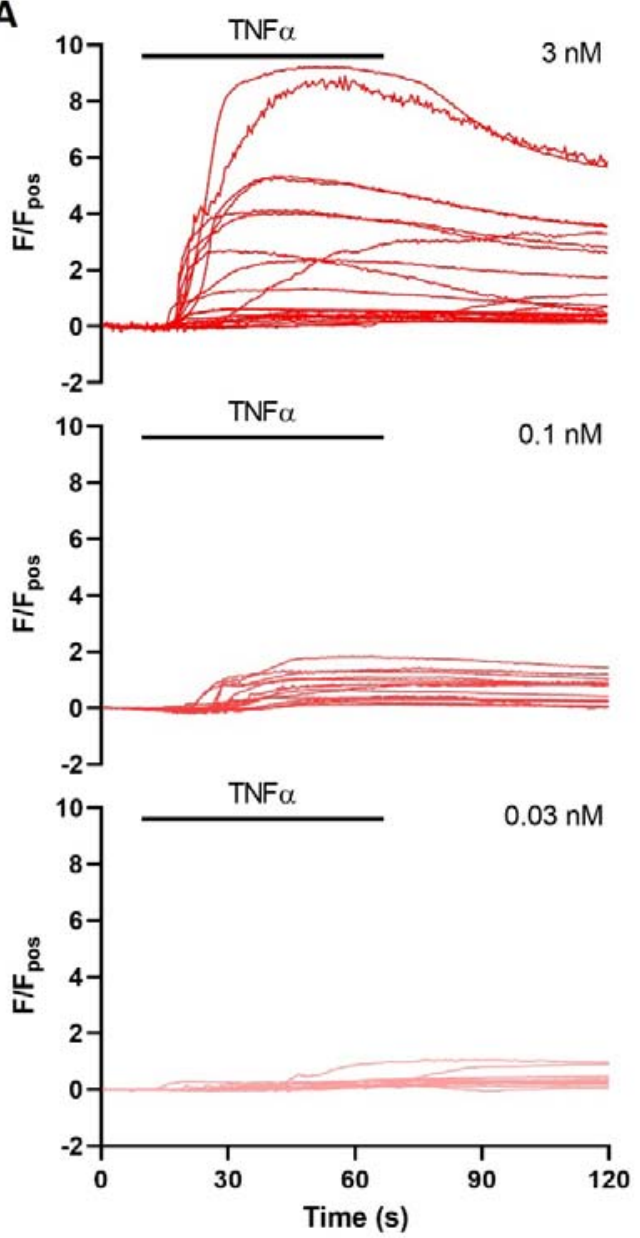

B

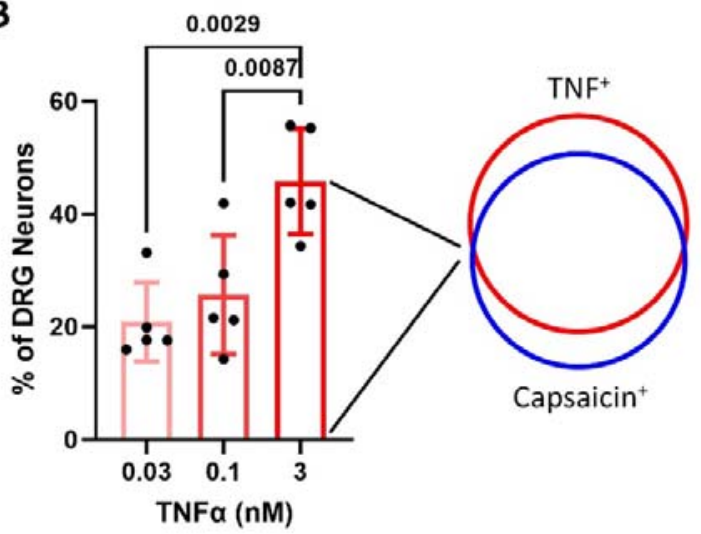

C
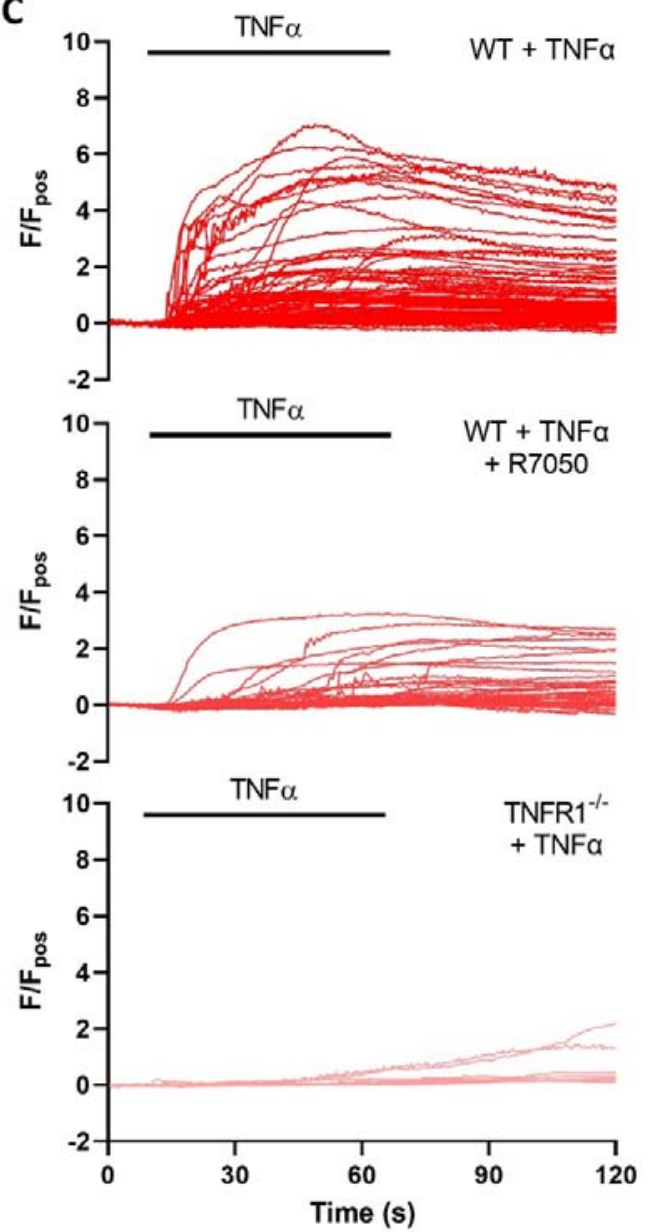

D

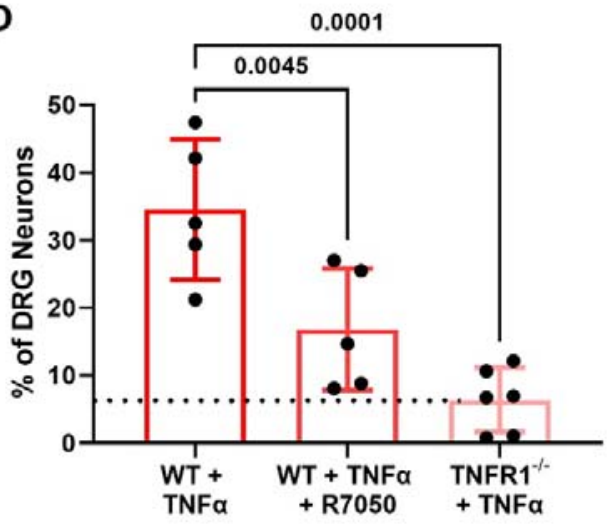

Figure 2 - Characterisation of TNFa-evoked $\left[\mathrm{Ca}^{2+}\right]_{i}$ increase in DRG neurons. (A) Representative traces illustrating the concentration-dependent increase in the magnitude of the normalised fluorescent response to TNFa (0.03 - $3.0 \mathrm{nM})$ within individual neurons from respective culture dishes. (B) The concentration-dependent increase in the proportion (per dish) of TNFa responsive DRG neurons ( $p=0.0022$, one-way ANOVA with Holm-Šídák's multiple comparisons test; $n=5$ dishes from $N=5$ independent cultures), of which the 
majority were also co-sensitive to capsaicin. The effects of TNFa (0.1nM) were TNFR1mediated as illustrated by the significant reduction in (C) response magnitude and (D) proportion of TNFa-responsive neurons following TNFR1 inhibition with R7050 (10 $\mu \mathrm{M})$ or genetic deletion of TNFR1 in Tnfrsf1a ${ }^{-/-}$mice in comparison to neurons from wild type (WT) animals ( $p=0.0003$, one-way ANOVA with Holm-Šídák's multiple comparisons test; $n=5-6$ dishes from $N=5-6$ independent cultures). Dotted line represents the proportion of neurons activated in ECS controls $(5.54 \pm 5.01 \%, n=6, N=6)$.

Further experiments revealed that the TNFa-mediated increase in $\left[\mathrm{Ca}^{2+}\right]_{\mathrm{i}}$ was lost following removal of extracellular $\mathrm{Ca}^{2+}$, but unaffected by depletion of intracellular $\mathrm{Ca}^{2+}$ stores, demonstrating that the rise in $\left[\mathrm{Ca}^{2+}\right]_{i}$ was driven by external $\mathrm{Ca}^{2+}$ entry $(p=0.00130$, oneway ANOVA with Holm-Šídák's multiple comparisons test; $n=5-8, N=5$; Figure $3 A$ ). Consistent with this observation, pre-treatment with the TRPV1 antagonist A425619 (1 $\mu \mathrm{M})$ significantly attenuated the proportion of neurons responding to TNFa $(p=0.0352$, unpaired t-test; $n=5, N=5$; Figure $3 B$ ) at a concentration that abolished responses to capsaicin (e.g. $4.39 \pm 2.29 \%$ in the presence of $A 425619$ vs $44.63 \pm 8.19 \%$ in the absence of A425619; $p<$ 0.0001 , unpaired t-test; $n=4-5, N=4-5$; data not shown). 
A

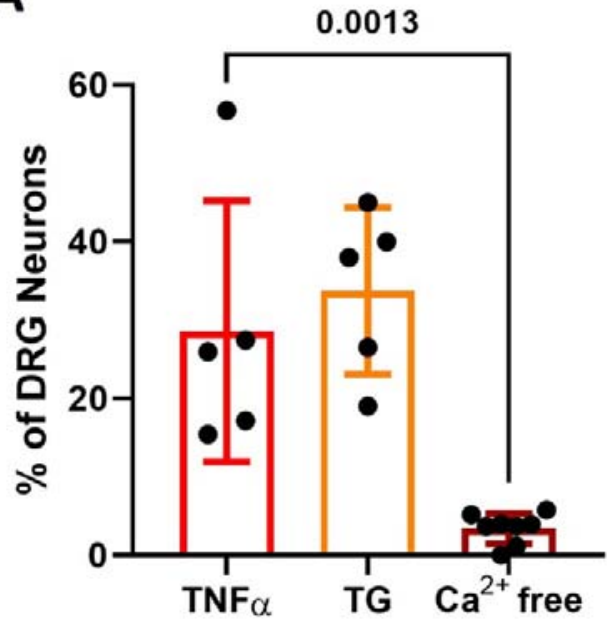

C

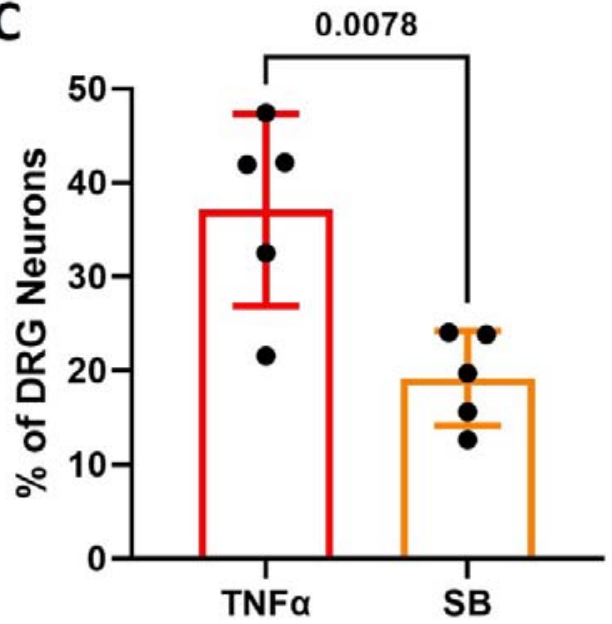

B

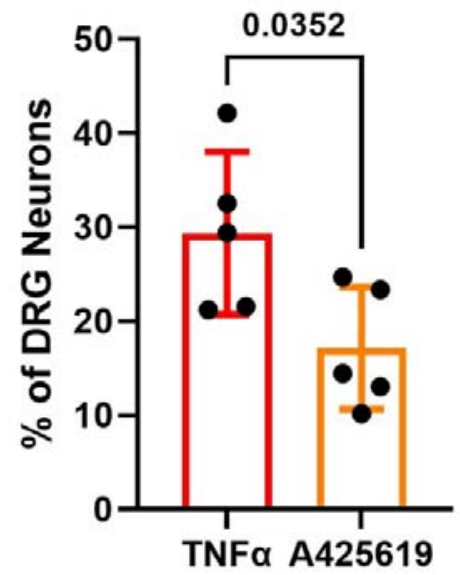

D

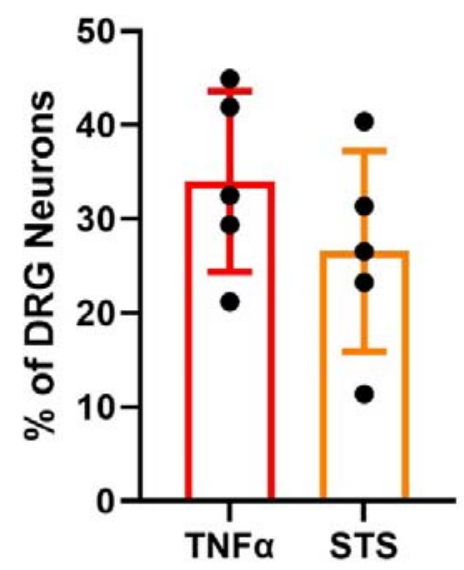

Figure 3 - TNFa-evoked $\left[\mathrm{Ca}^{2+}\right]_{i}$ increase is mediated by extracellular $\mathrm{Ca}^{2+}$, TRPV1 and p38 MAPK activation. (A) The proportion of DRG neurons responding to $0.1 \mathrm{nM} T N F \alpha$ was unchanged following depletion of internal $\mathrm{Ca}^{2+}$ stores with $1 \mu \mathrm{M}$ thapsigargin (TG) and abolished by removal of external $\mathrm{Ca}^{2+}(p=0.0002$, one-way ANOVA; $n=5-8$ dishes from $N$ $=5$ independent cultures). This effect was partially mediated by TRPV1 channels as illustrated $(B)$ by the significant reduction in $T N F \alpha$-sensitive neurons following pre-incubation with TRPV1 inhibitor $1 \mu M$ A425619 ( $p=0.0352$, unpaired $t$-test; $n=5$ dishes from $N=5$ independent cultures). Furthermore, $(C \& D)$ the proportion of neurons activated by TNFa was significantly attenuated following p38 MAPK inhibition with $10 \mu \mathrm{M}$ SB203580 (SB) ( $p=$ 0.0078 , unpaired $t$-test; $n=5$ dishes from $N=5$ independent cultures), but not inhibition of protein kinase $C$ (PKC) activity with $10 \mu \mathrm{M}$ staurosporine (STS; $p=0.283$, unpaired $t$-test; $n$ $=5$ dishes from $N=5$ independent cultures). 
In addition, pre-treatment with the p38 MAPK inhibitor SB203580 (10 $\mu \mathrm{M})$, but not the protein kinase $\mathrm{C}(\mathrm{PKC})$ inhibitor staurosporine $(10 \mu \mathrm{M})$, significantly reduced the proportion of TNFa-sensitive neurons ( $p=0.00780$, unpaired t-test; $n=5, N=5$; Figure $3 C \& D$ ) in agreement with previous findings demonstrating a role for p38 MAPK in TNFa-mediated neuronal sensitisation (Gudes et al., 2015). Furthermore, in ultrapure DRG cultures, in which non-neuronal cells were removed by MACS $(p<0.0001$, unpaired t-test; $n=3, N=3$; Figure $4 A \& B$ ), the response to TNFa was still observed in a comparable proportion of neurons ( $p$ $=0.491$, unpaired t-test; $n=4-5, N=4-5$ ), thereby confirming that TNFa can directly stimulate sensory neurons, consistent with reported TNFR1 expression in DRG neurons (Figure 4C \& D).

A
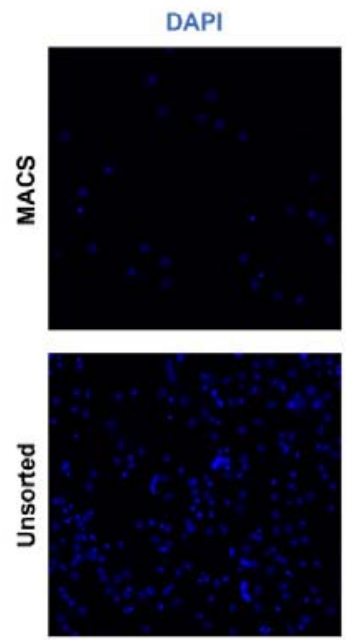

C

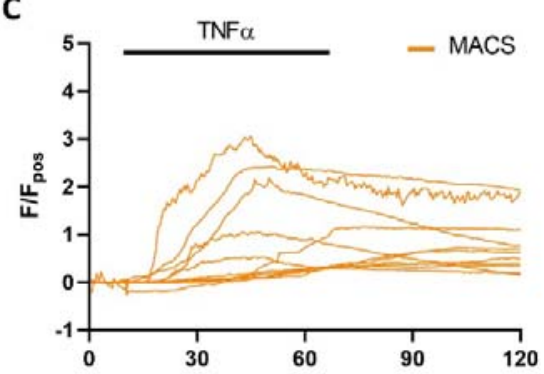

$\beta I I I-t u b$
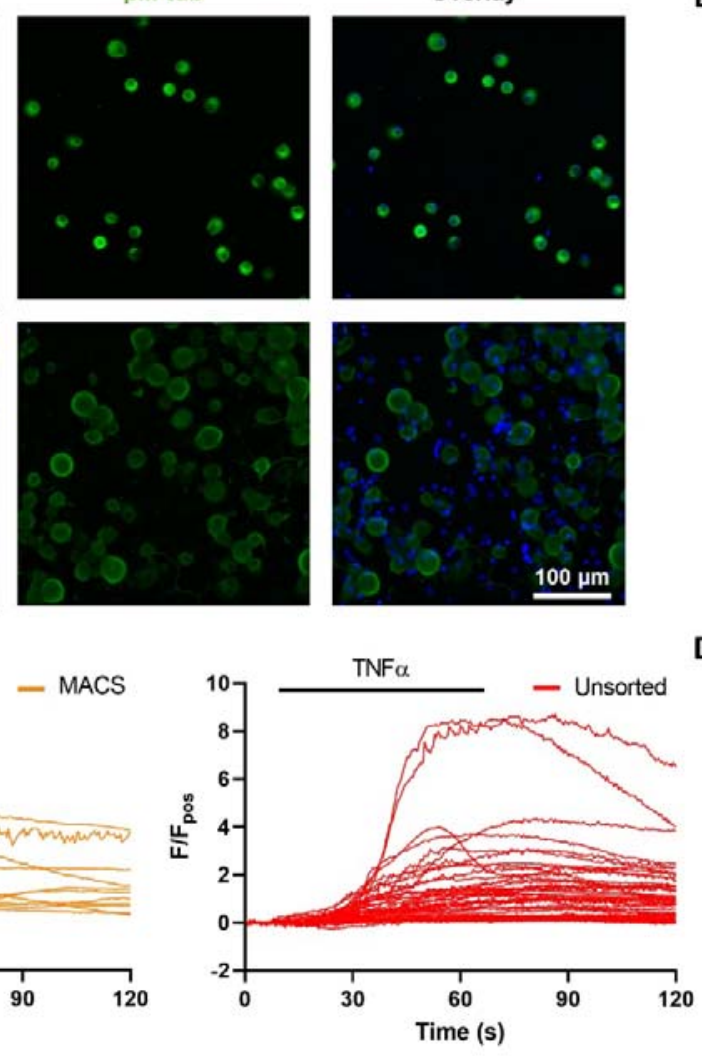

B
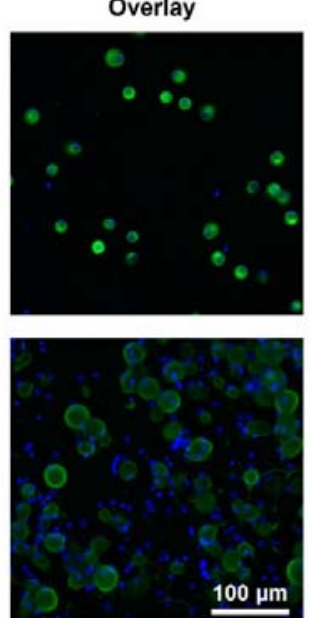

D

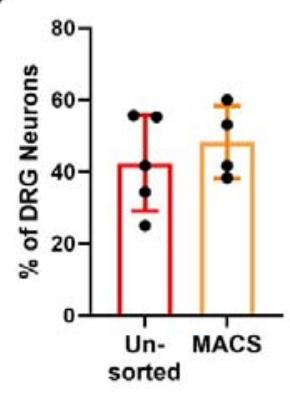

Figure 4 - TNFa acts directly on DRG neurons. Magnetic-activated cell sorting (MACS) was used to generate ultra-pure neuronal cultures as illustrated by $(A)$ the immunofluorescent staining of $\beta$ III tubulin (green) and DAPI (blue) in respective cultures and (B) the greatly increased proportion of cells stained with Blll tubulin following MACS sorting ( $p<0.0001$, unpaired t-test; $n=3$ dishes from $N=3$ independent cultures). TNFa mediated robust increases in $\left[\mathrm{Ca}^{2+}\right]_{i}$ in ultra-pure and unsorted cultures illustrated (C) by individual traces from respective cultures and confirmed (D) by the comparable percentage of TNFa 
responders in unsorted and MACS sorted DRG cultures ( $p=0.290$, unpaired t-test; $n=4-5$ dishes from $N=4-5$ independent cultures).

Having established the important contribution of p38 MAPK signalling and TNFR1 expression to TNFa-mediated $\mathrm{Ca}^{2+}$ flux, we next confirmed the involvement of this pathway in the sensitisation of TRPV1 signalling by TNFa. No sensitisation of the magnitude of the $\left[\mathrm{Ca}^{2+}\right]_{\mathrm{i}}$ response to capsaicin following 24-hour incubation with TNFa was observed in tissue from TNFR $1^{-1}$ mice ( $p=0.787$, unpaired t-test; $n=6, N=6$; Figure $5 A \& B$ ), and the proportion of capsaicin-sensitive DRG neurons was comparable to controls $(p=0.891$, unpaired t-test; $\mathrm{n}=6, \mathrm{~N}=6$; Figure $5 \mathrm{C}$ ). Following co-incubation of TNFa with SB203580, TNFa-mediated sensitisation of capsaicin responses was attenuated ( $p=0.736$, unpaired ttest; $n=5, N=5$; Figure 5D \& E), and the proportion of responders unchanged ( $p=0.907$, unpaired t-test; $n=5, N=5$; Figure $5 F$ ). 

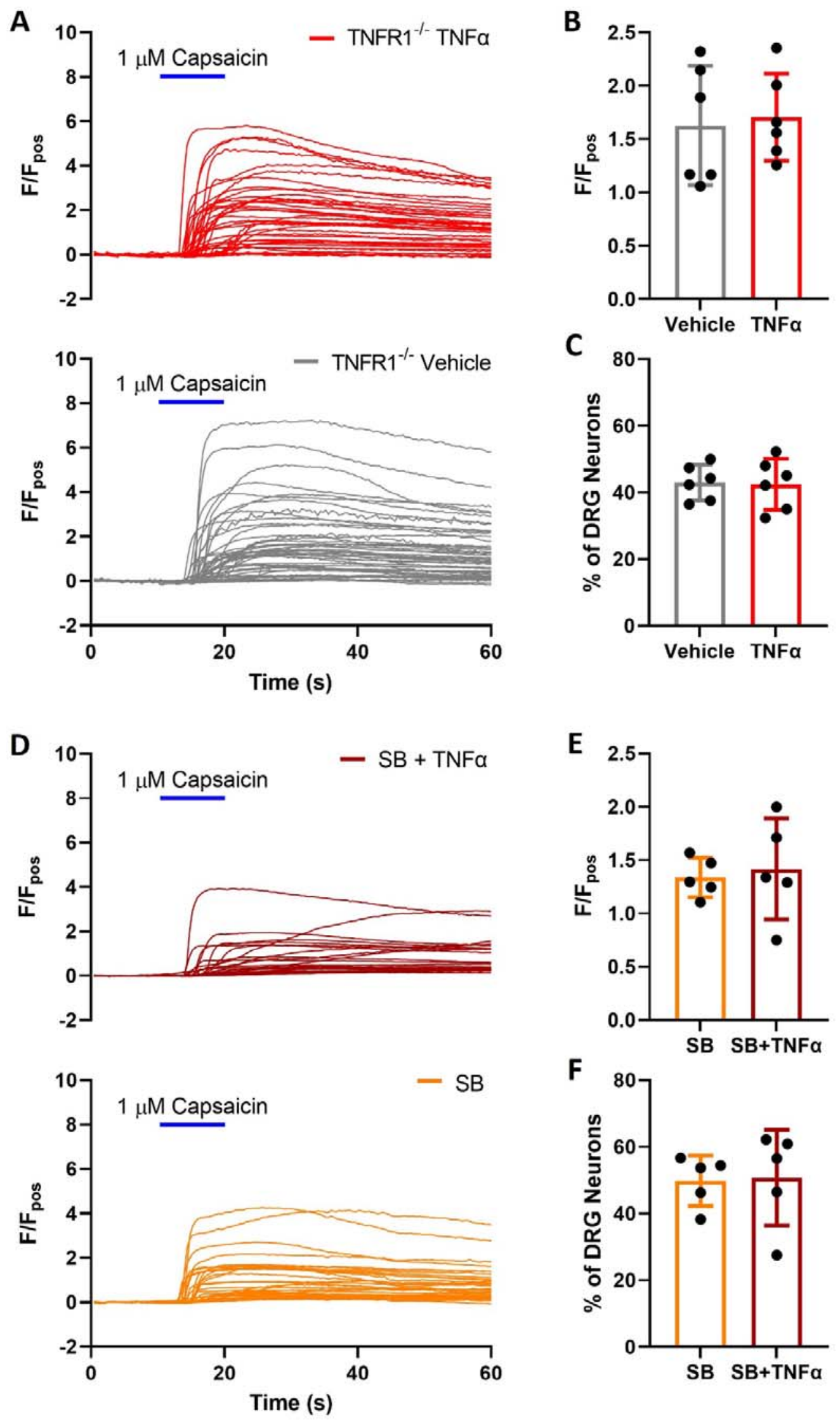
Figure 5 - TNFa sensitisation of capsaicin-evoked $\left[\mathrm{Ca}^{2+}\right]_{i}$ increase in DRG neurons is p38 MAPK- and TNFR1-mediated. (A) Example traces of individual response profiles to $1 \mu \mathrm{M}$ capsaicin (10 s) in DRG neurons cultured from TNFR1\% mice following 24-hour treatment of respective culture dishes with vehicle (PBS) or TNFa (3 nM). (B) Incubation with TNFa no longer increased peak averaged per dish $\mathrm{Ca}^{2+}$ responses to capsaicin in DRG neurons from TNFR $1^{-1}$ mice ( $p=0.787$, unpaired $t$-test; $n=6$ dishes from $N=6$ independent cultures). (C) The proportion of capsaicin-sensitive neurons remained comparable between culture dishes pre-incubated with vehicle or TNFa in DRG neurons from TNFR1 ${ }^{-1-}$ mice $(p=0.891$, unpaired t-test; $n=6$ dishes from $N=6$ independent cultures). Similarly, overnight incubation with TNFa no longer increased the magnitude of capsaicin responses in DRG neurons coincubated with the p38 MAPK inhibitor SB203580 as illustrated by: (D) individual neuronal responses to $1 \mathrm{\mu M}$ capsaicin (10 s) in SB203580-treated DRG neurons following $24 \mathrm{~h}$ incubation with vehicle or TNFa (3 nM); (E) the comparable magnitude of the peak averaged per dish response to capsaicin between respective treatments $(p=0.736$, unpaired t-test; $n$ = 5 dishes from $N=5$ independent cultures). ( $F$ ) The proportion of capsaicin-sensitive $D R G$ neurons was also comparable between treatment groups following p38 MAPK inhibition ( $p=$ 0.907, unpaired $t$-test; $n=5$ dishes from $N=5$ independent cultures).

\section{TNFa sensitises colonic afferent responses to noxious ramp distension and capsaicin via TNFR1 and p38 MAPK}

Finally, to confirm the translation of our findings from DRG neurons to the activation of colonic afferents, we studied the contribution of TNFR1 and p38 MAPK signalling to TNFamediated sensitisation of colonic afferent responses to noxious ramp distension and capsaicin (Figure 6A). Consistent with the sensitising effects of TNFa observed previously in DRG neurons, treatment with TNFa prevented the desensitisation of colonic afferent responses to repeated ramp distensions $(p=0.0004$, two-way ANOVA with Holm-Šídák's multiple comparisons test; $N=8$; Figure $6 \mathrm{~B}$ ). This resulted in significantly greater afferent responses to ramp distension across noxious distending pressures $(p=0.0002$, multiple unpaired t-tests, $N=8$; Figure $6 \mathrm{C}$ ) and significantly increased peak afferent firing ( $p=$ 0.0004 , unpaired t-test, $N=8$; Figure $6 \mathrm{D}$ ). An enhanced afferent response to capsaicin was also observed following application of TNFa compared with vehicle $(p=0.0138$, unpaired $t-$ test; $N=8$; Figure 7A \& B). TNFa significantly increased nerve firing in the 10 minutes post capsaicin application ( $p=0.0049$, two-way ANOVA with Holm-Šídák's multiple comparisons test; $N=8$; Figure 7C) and peak activity was elevated 75\% compared to vehicle ( $p=0.0069$, unpaired t-test, $\mathrm{N}=8$; Figure 7D). Compliance of distensions was comparable between treatment groups (e.g. $0.741 \pm 0.137 \mathrm{ml}$ vs $0.731 \pm 0.074 \mathrm{ml}$ for the 5 th distension in TNFa vs vehicle treated tissues, $\mathrm{p}=0.8421$, unpaired t-test; $\mathrm{N}=8$; data not shown). 


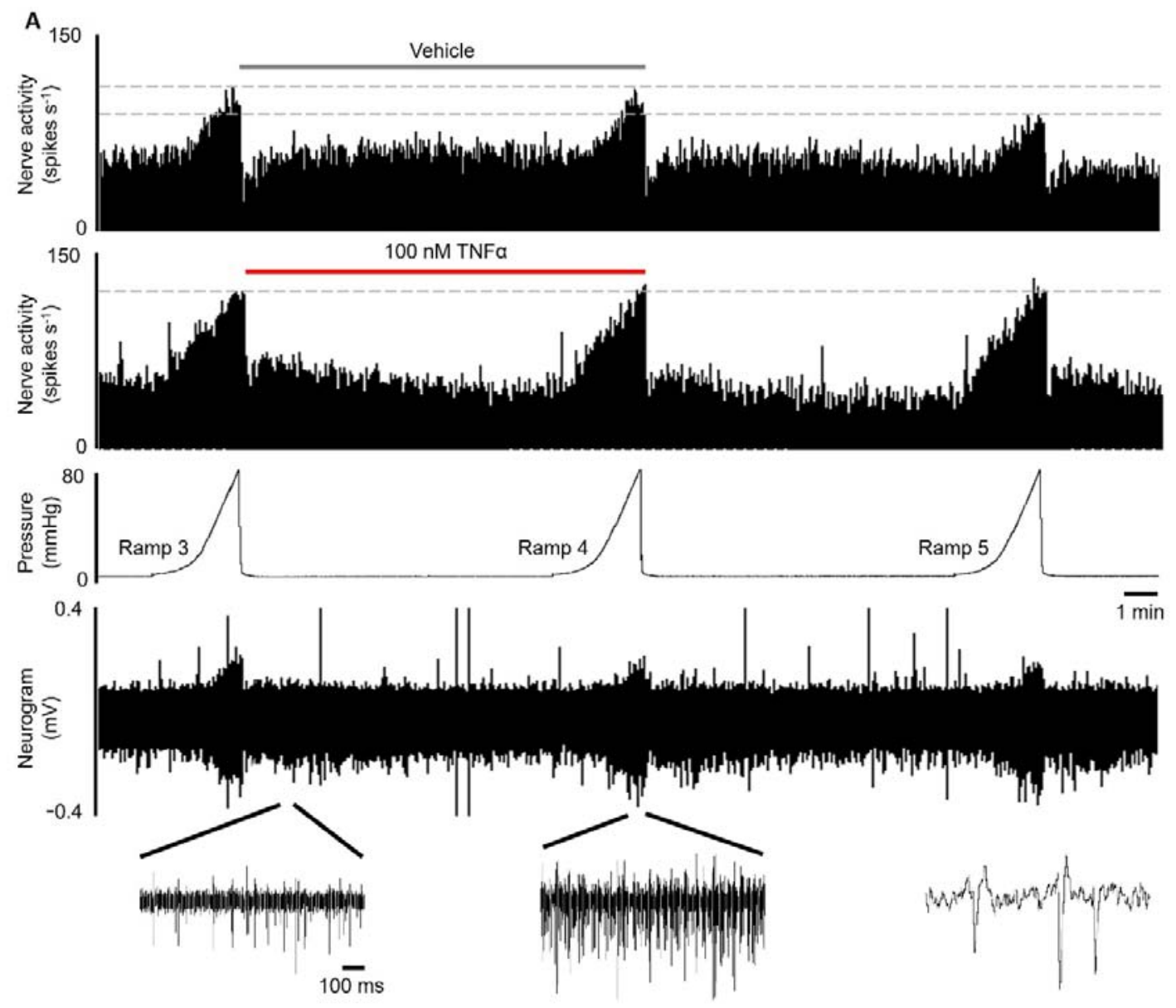

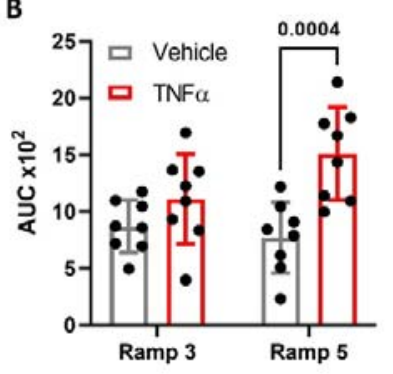

C

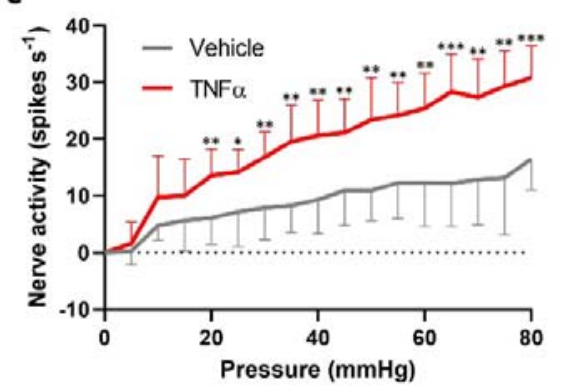

D

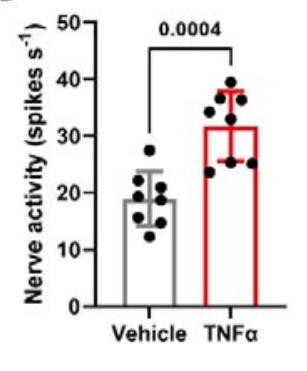

Figure 6 - TNFa evokes colonic afferent mechanical hypersensitivity. (A) Example rate histograms and neurogram of lumbar splanchnic nerve (LSN) activity with accompanying pressure trace showing sequential $(x 3)$ ramp distensions $(0-80 \mathrm{mmHg})$ from vehicle- and TNFa (100 nM)-treated preparations, highlighting the desensitisation of afferent responses to distension no longer occurred following TNFa treatment. This effect was confirmed by: $(B)$ the significantly greater afferent response to ramp distension (measured by area under the curve, AUC, during ramp distension) following TNFa pre-treatment compared with vehicle (ramp 5) ( $p=0.0004$, two-way ANOVA, $N=8)$; (C) the significantly greater increase in afferent response throughout the noxious distending pressure range $(<20 \mathrm{mmHg}$, ramp 5) 
following TNF $\alpha$ treatment compared with vehicle $\left({ }^{*} p<0.05,{ }^{* *} p<0.01,{ }^{* * *} p<0.001\right.$, multiple t-tests; $N=8$ animals); (D) the significantly greater peak increase in afferent discharge to ramp distension following application of TNF $\alpha$ compared with vehicle $(p=$ 0.0004, unpaired t-test; $N=8$ ).

A
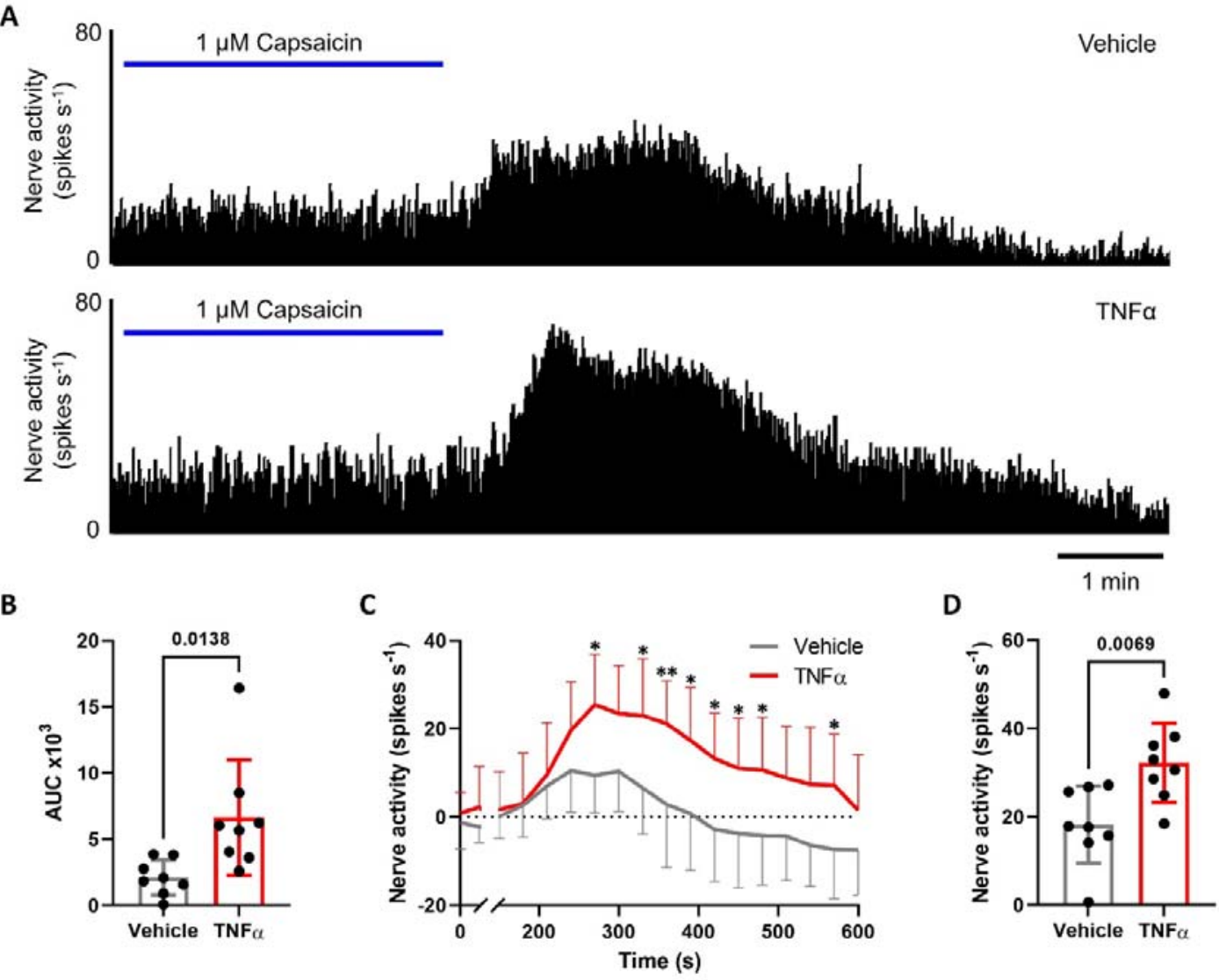

Figure 7 - TNFa sensitises colonic afferent responses to capsaicin. (A) Example rate histograms of $L S N$ recordings illustrating the greater afferent response to $1 \mu M$ capsaicin following TNFa (100 nM) compared with vehicle pre-treatment. This was confirmed by the significantly greater afferent discharge following TNF $\alpha$ compared with vehicle pre-treatment measured by: (B) AUC of afferent firing 0-10 min post capsaicin application $(p=0.0138$, unpaired t-test; $N=8$ animals); (C) prolonged increase in afferent discharge to capsaicin $\left({ }^{*} p\right.$ $<0.05$, ${ }^{* *} p<0.01$, two-way ANOVA with Holm-Šídák's multiple comparisons test; $N=8$ animals); (D) greater peak change in afferent firing 0-10 min after application of capsaicin ( $p$ $=0.0042$, unpaired $t$-test; $N=8$ animals).

In keeping with data from DRG neurons, where we observed a reliance on TNFR1 for the sensitising effects of TNFa, no sensitisation of responses to noxious ramp distension was observed following the application of TNFa compared with vehicle in tissue from TNFR $1^{-1-}$ mice ( $p=0.5209$, unpaired t-test; $N=7-8$; Figure $8 A$ ). In addition, no difference in afferent 
activity was seen across noxious distending pressures $(p=0.2803$, multiple unpaired t-tests, $\mathrm{N}=7-8$; Figure $8 \mathrm{~B}$ ) and peak afferent firing was unchanged between vehicle- and TNFatreated TNFR $1^{-/}$tissue ( $\mathrm{p}=0.6658$, unpaired t-test, $\mathrm{N}=7-8$; Figure $8 \mathrm{C}$ ). No TNFa-mediated sensitisation was observed in response to capsaicin ( $p=0.7668$, unpaired t-test; $N=7-8$; Figure 8D), and afferent firing was comparable 10 minutes after capsaicin application ( $p=$ 0.9611, two-way ANOVA with Holm-Šídák's multiple comparisons test; $\mathrm{N}=7-8$; Figure 8E). Furthermore, TNFa had no effect on peak afferent activity in the absence of TNFR1 $(p=$ 0.9671 , unpaired t-test, $N=7-8$; Figure $8 F$ ). Interestingly, responses to ramp distension (AUC: WT $769 \pm 312$ vs TNFR1 ${ }^{-1} 1665 \pm 412 ; p=0.0004$, unpaired t-test; $N=7-8$ ) and capsaicin (AUC: WT $2114 \pm 1335$ vs TNFR1 ${ }^{-/} 5615 \pm 2535 ; \mathrm{p}=0.0046$, unpaired t-test; $\mathrm{N}=$ 7-8) were significantly higher in vehicle treated tissues from TNFR $1^{-/}$mice compared to wild type (WT) mice.

A

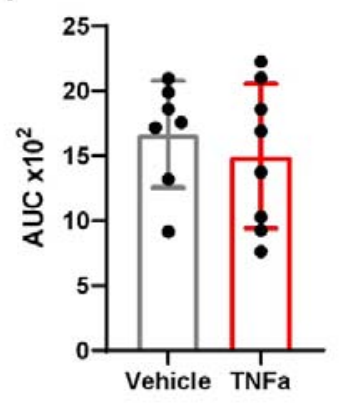

D

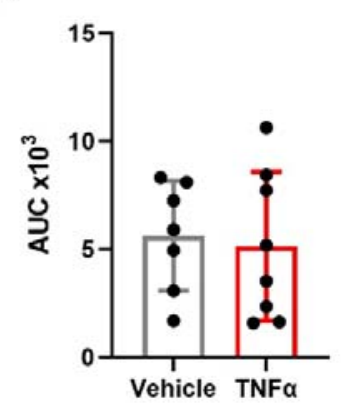

B

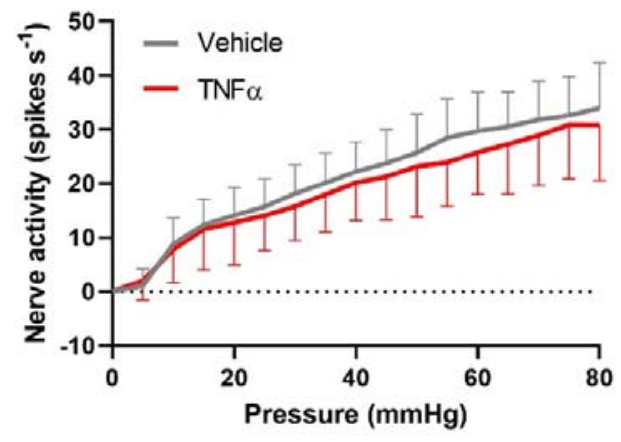

E

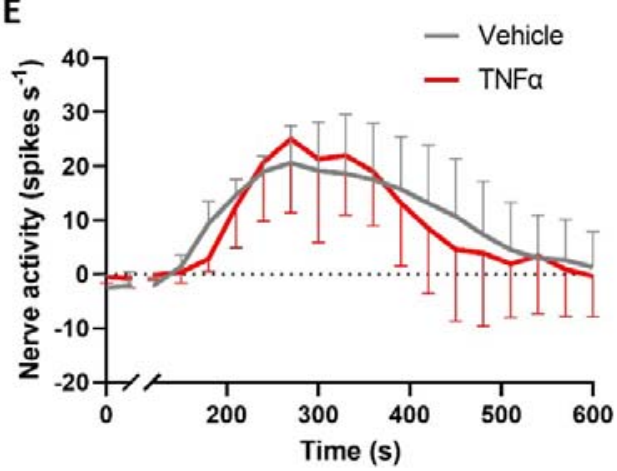

C

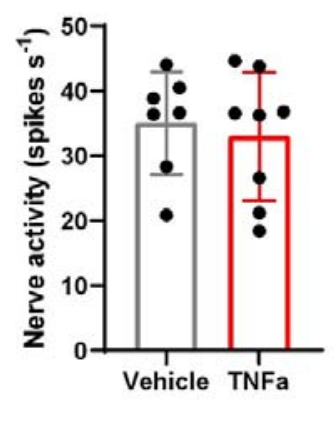

$\mathbf{F}$

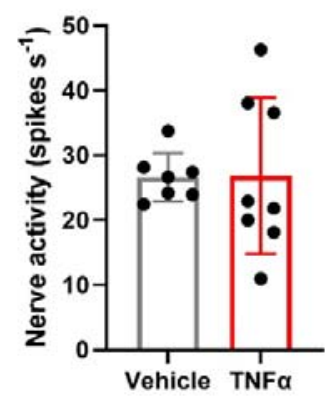

Figure 8 - TNFR1 mediates TNFa-induced colonic afferent sensitisation. The sensitisation of colonic afferents by TNFa pre-treatment was dependent on TNFR1 expression as confirmed by the presence of comparable colonic afferent responses to ramp distension and capsaicin following vehicle or TNFa pre-treatment in tissue from TNFR $1^{-1}$ mice shown by: (A) comparing the AUC of nerve activity following ramp distension ( $p=0.5209$, unpaired $t$ test; $N=7-8)$; (B) afferent response profiles to ramp distension ( $p=0.280$, multiple t-tests; $N$ 
= 7-8 animals); (C) peak firing frequency to ramp distension ( $p=0.6658$, unpaired t-test; $N=$ 7-8); (D) AUC of firing frequency following capsaicin application ( $p=0.7668$, unpaired $t$-test; $N=7-8) ;(E)$ afferent response profiles to capsaicin ( $p=0.9611$, two-way ANOVA with HolmŠídák's multiple comparisons test; $N=7-8$ animals); $(F)$ the peak increase in afferent discharge to capsaicin ( $p=0.967$, unpaired t-tests; $N=7-8$ ).

Having identified a role for p38 MAPK signalling in the sensitising effects of TNFa in DRG neurons, we observed no sensitisation of colonic afferents to noxious ramp distension following the application of TNFa in the presence of p38 MAPK inhibition with SB203580 ( $p$ $=0.0148$, one-way ANOVA with Holm-Šídák's multiple comparisons test; $N=6-8$; Figure 9A). SB203580 attenuated TNFa sensitisation across all noxious distending pressures ( $p=$ 0.004340; multiple unpaired t-tests; $N=7-8$; Figure 9B) and p38 MAPK inhibition significantly decreased peak afferent firing following TNFa incubation ( $p=0.0211$, one-way ANOVA with Holm-Šídák's multiple comparisons test; $\mathrm{N}=6-8$; Figure $9 \mathrm{C}$ ). Furthermore, SB203580 significantly attenuated afferent responses to capsaicin in TNFa-treated tissue ( $p$ $=0.0349$, one-way ANOVA with Holm-Šídák's multiple comparisons test; $N=7-8$; Figure 9D \& E) and reduced peak afferent firing $(p=0.0321$, one-way ANOVA with Holm-Šídák's multiple comparisons test; $\mathrm{N}=7-8$; Figure $9 F$ ).

A

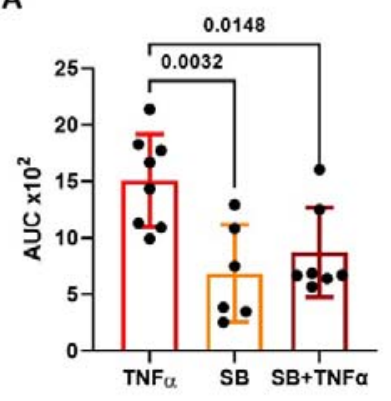

D

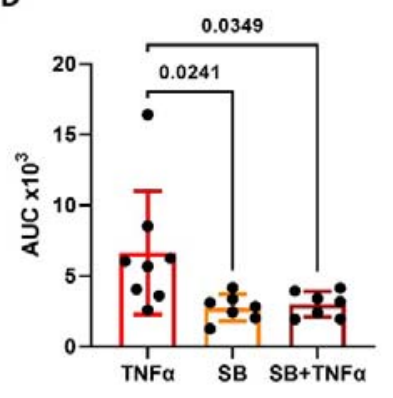

B

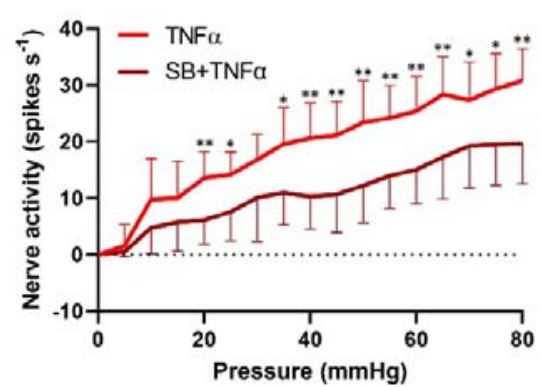

E

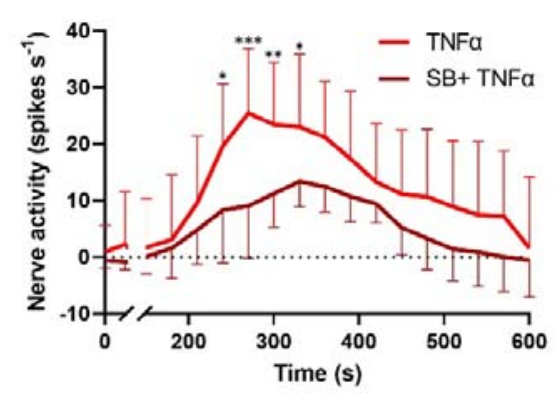

C

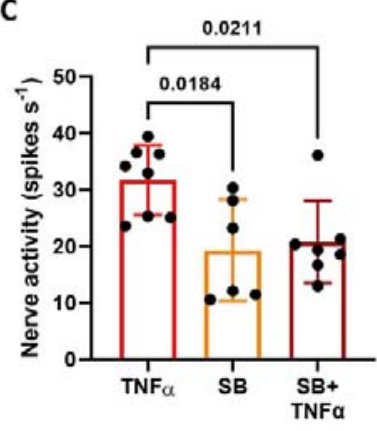

F

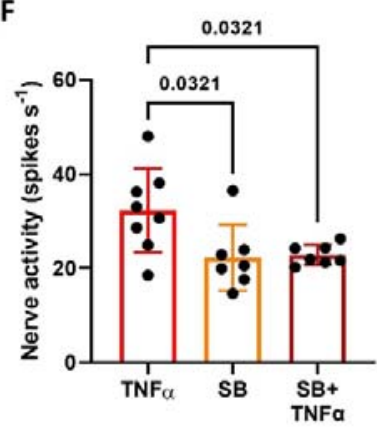

Figure 9 - TNFa-induced colonic afferent sensitisation is mediated by p38 MAPK. The ability of TNFa to sensitise colonic afferent responses to ramp distension and capsaicin was 
abolished by co-administration with the p38 MAPK inhibitor SB203589 as demonstrated by: (A) the significant reduction in AUC of colonic afferent responses to ramp distensions following pre-treatment with TNFa in the presence of SB203589 ( $p=0.0034$, one-way ANOVA with Holm-Šídák's multiple comparisons test; $N=6-8$ animals); (B) the significant reduction in afferent responses to ramp distension across noxious distending pressures (< $20 \mathrm{mmHg}$ ) following pre-treatment with TNF $\alpha$ in the presence of SB203580 $\left(^{*} p<0.05,{ }^{* *} p<\right.$ 0.01, multiple t-tests; $N=6-8$ animals); (C) the significant reduction in the peak afferent response to ramp distension following application of TNF $\alpha$ in the presence of SB203580 ( $p=$ 0.0092, one-way ANOVA with Holm-Šídák's multiple comparisons test; $N=6$-8 animals); (D) significantly reduced afferent responses to capsaicin measured by AUC following pretreatment with TNF $\alpha$ in the presence of SB203580 ( $p=0.0202$, one-way ANOVA with HolmŠídák's multiple comparisons test; $N=7-8$ animals); (E) the significant reduction in afferent responses to capsaicin at multiple time points following pre-treatment with TNFa in the presence of SB203580 $\left({ }^{*} p<0.05,{ }^{* *} p<0.01,{ }^{* *} p<0.001\right.$, two-way ANOVA with HolmŠídák's multiple comparisons test; $N=7-8$ animals); ( $F)$ the significant reduction in peak nerve discharge to capsaicin following pre-treatment with TNFa in the presence of SB203580 ( $p=0.0162$, one-way ANOVA Holm-Šídák's multiple comparisons test; $N=7-8$ animals). 


\section{Discussion}

TNFa has been linked to the production of abdominal pain in gastrointestinal disease due to its enhanced expression in disease states such as IBS (Hughes et al., 2013) and IBD (Kamada et al., 2008), combined with data showing TNFa-mediated visceral nociceptor sensitisation and TNFR1 expression in colonic nociceptors (Hockley et al., 2019). The signalling pathways mediating these effects have not been fully explored, although data from studies of somatic hypersensitivity point to a role for p38 MAPK signalling downstream of TNFR1 receptor activation (Jin \& Gereau IV, 2006) and a sensitising effect on TRPV1, a detector of noxious stimuli (Khan et al., 2008). As such, the goal of this study was to investigate the contribution of TNFR1 and p38 MAPK signalling to the pro-nociceptive effects of TNFa including TRPV1 receptor activation in sensory neurons and colonic afferents.

Data from our studies confirmed that TNFa sensitises TRPV1 receptor signalling, by demonstrating: i) an enhanced capsaicin-evoked increase in $\left[\mathrm{Ca}^{2+}\right]_{i}$ within sensory neurons following overnight incubation with TNFa; ii) a TRPV1 receptor-mediated increase in $\left[\mathrm{Ca}^{2+}\right]_{i}$ to acute application of TNFa in sensory neurons that subsequently showed significantly less desensitisation to repeated application of capsaicin; and iii) a marked increase in the colonic afferent response to capsaicin following TNFa pre-treatment. In keeping with our hypothesis, these effects were no longer observed in tissue from TNFR $1^{-1-}$ mice and were greatly attenuated by pre-treatment with the p38 MAPK inhibitor SB203580; conditions in which TNFa also no longer prevented the desensitisation of colonic afferent responses to repeated noxious distension of the bowel. Collectively these findings provide further functional evidence of a contribution by TNFa to the production of visceral pain in gastrointestinal disease and highlight the role of TNFR1-mediated p38 MAPK signalling to the pronociceptive activity of TNFa.

The validity of our data is supported by comparable observations showing TNFa enhances TRPV1 receptor signalling in nodose (Hsu et al., 2017; Lin et al., 2017), trigeminal (Khan et al., 2008) and DRG neurons (Hensellek et al., 2007). These effects have been attributed to TNFR1 activation and p38 MAPK signalling, consistent with our data demonstrating the essential contribution of TNFR1 and p38 MAPK to TNFa sensitisation of capsaicin responses in colonic afferents for the first time, and DRG neurons for the first time in the same study. The mechanism by which p38 MAPK modulates TRPV1 receptor activity was not a focus of our experiments, however we observed changes following brief application of TNFa indicative of an acute increase in channel activity that would be expected following phosphorylation of TRPV1 by p38 MAPK. This observation was in keeping with previous studies showing enhanced TRPV1-mediated inward currents shortly after application of 
TNFa; effects reported to be mediated by p38 MAPK and protein kinase C (PKC) (Constantin et al., 2008). However in contrast to p38 MAPK inhibition, we found no change in the TRPV1-mediated increase in $\left[\mathrm{Ca}^{2+}\right]_{i}$ to TNFa following PKC inhibition with staurosporine. These findings suggest that phosphorylation sites on intracellular domains of TRPV1, which are not specific to PKC, may be targeted and phosphorylated by p38 MAPK. In addition, p38 MAPK signalling has also been shown to increase TRPV1 channel expression following incubation with TNFa for periods of 1 hour or greater (Constantin et al., 2008), and this process may also have contributed to the increased capsaicin response we observed following overnight incubation with TNFa.

Consistent with the reported co-expression of TNFR1 with TRPV1 in sensory neurons (Zeisel et al., 2018), we found marked co-sensitivity of TNFa-stimulated DRG neurons to capsaicin. This response was dependent on TNFR1 expression and was preserved in ultrapure neuron specific DRG cultures demonstrating that TNFa can directly modulate sensory neuron activity and TRPV1 signalling via TNFR1 in the same neuron. Furthermore, these data indicate that TNFa-responsive neurons are predominantly nociceptors based on the ability of capsaicin to evoked significant pain in humans, including visceral pain following colorectal administration (van Wanrooij et al., 2014). This observation was corroborated by our finding that TNFa sensitises colonic afferents at noxious distending pressures. These experiments provide evidence that TNFa causes abdominal pain through TNFR1-mediated colonic nociceptor sensitisation due to p38 MAPK-enhanced TRPV1 signalling.

However, this is not the only mechanism by which TNFa stimulates sensory neurons. For example, the increase in $\left[\mathrm{Ca}^{2+}\right]_{i}$ to TNFa, which is solely dependent on extracellular $\mathrm{Ca}^{2+}$ entry, was only partially blocked by the TRPV1 antagonist (A425619) at concentrations that completely abolished the $\mathrm{Ca}^{2+}$ response to capsaicin. This indicates that other $\mathrm{Ca}^{2+}$ permeable ion channels are stimulated by TNFa consistent with existing data implicating TRPA1 in TNFa-mediated afferent sensitisation (Hughes et al., 2013). In addition, we also demonstrated that TNFa-mediated colonic afferent mechanosensitisation was dependent on TNFR1 expression and p38 MAPK activity in keeping with seminal data showing this signalling pathway mediates somatic mechanical hypersensitivity to TNFa (Jin \& Gereau IV, 2006). These effects have been attributed to enhanced TTX-resistant Nav1.8 currents, an effect also seen in response to TNFR1 dependent TNFa signalling in colon projecting DRG neurons. With the inclusion of our study, current data indicates that TNFa has the capacity to sensitise colonic nociceptors through p38 MAPK-enhanced TRPV1, TRPA1 and Nav1.8 channel activity downstream of TNFR1. 
These findings highlight the utility of TNFa and its downstream signalling pathways as important drug targets for pain relief in gastrointestinal diseases, such as IBS and IBD, in which enhanced TNFa expression has been reported.

\section{Additional Information}

\section{Data availability}

Raw data sets can be found at DOI: $10.17863 /$ CAM.81112

\section{Competing Interests}

F.W. and I.P.C. are employed by AstraZeneca.

\section{Author Contributions}

K.H.B. designed the research studies, conducted the experiments, acquired and analysed the data and wrote the manuscript. J.P.H. acquired and analysed the data and wrote the manuscript. T.S.T. acquired the data. L.A.P. analysed the data. I.P.C. designed the research studies. DCB, E.St.J.S. and FW designed the research studies and wrote the manuscript. All authors approved the final version of the manuscript submitted for publication.

\section{Funding}

This work was supported by AstraZeneca PhD Studentship (K.H.B.), and the University of Cambridge BBSRC Doctoral Training Program (J.P.H.; L.A.P.: BB/M011194/1). 


\section{References}

Constantin, C. E., Mair, N., Sailer, C. A., Andratsch, M., Xu, Z. Z., Blumer, M. J. F., Scherbakov, N., Davis, J. B., Bluethmann, H., Ji, R. R., \& Kress, M. (2008).

Endogenous tumor necrosis factor a (TNFa) requires TNF receptor type 2 to generate heat hyperalgesia in a mouse cancer model. Journal of Neuroscience, 28(19), 50725081. https://doi.org/10.1523/JNEUROSCI.4476-07.2008

Crerar, H., Scott-Solomon, E., Bodkin-Clarke, C., Andreassi, C., Hazbon, M., Logie, E., Cano-Jaimez, M., Gaspari, M., Kuruvilla, R., \& Riccio, A. (2019). Regulation of NGF Signaling by an Axonal Untranslated mRNA. Neuron, 102(3), 553. https://doi.org/10.1016/J.NEURON.2019.02.011

Edelstein, A. D., Tsuchida, M. A., Amodaj, N., Pinkard, H., Vale, R. D., \& Stuurman, N. (2014). Advanced methods of microscope control using $\mu$ Manager software. Journal of Biological Methods, 1(2), e10. https://doi.org/10.14440/jbm.2014.36

Grundy, L., Erickson, A., \& Brierley, S. M. (2019). Visceral Pain. Annual Review of Physiology, 81, 261-284. https://doi.org/10.1146/annurev-physiol-020518-114525

Gudes, S., Barkai, O., Caspi, Y., Katz, B., Lev, S., \& Binshtok, A. M. (2015). The role of slow and persistent ttx-resistant sodium currents in acute tumor necrosis factor-a-mediated increase in nociceptors excitability. Journal of Neurophysiology, 113(2), 601-619. https://doi.org/10.1152/jn.00652.2014

Hensellek, S., Brell, P., Schaible, H. G., Bräuer, R., \& Segond von Banchet, G. (2007). The cytokine TNFa increases the proportion of DRG neurones expressing the TRPV1 receptor via the TNFR1 receptor and ERK activation. Molecular and Cellular Neuroscience, 36(3), 381-391. https://doi.org/10.1016/j.mcn.2007.07.010

Hockley, J. R. F., Taylor, T. S., Callejo, G., Wilbrey, A. L., Gutteridge, A., Bach, K., Winchester, W. J., Bulmer, D. C., McMurray, G., \& Smith, E. S. J. (2019). Single-cell RNAseq reveals seven classes of colonic sensory neuron. Gut, 68(4), 633-644. https://doi.org/10.1136/gutjnl-2017-315631

Hsu, C. C., Lin, Y. S., Lin, R. L., \& Lee, L. Y. (2017). Immediate and delayed potentiating effects of tumor necrosis factor-a on TRPV1 sensitivity of rat vagal pulmonary sensory neurons. American Journal of Physiology - Lung Cellular and Molecular Physiology, 313(2), L293-L304. https://doi.org/10.1152/ajplung.00235.2016

Hughes, P. A., Brierley, S. M., Martin, C. M., Brookes, S. J. H., Linden, D. R., \& Blackshaw, L. A. (2009). Post-inflammatory colonic afferent sensitisation: Different subtypes, different pathways and different time courses. Gut, 58(10), 1333-1341. https://doi.org/10.1136/gut.2008.170811

Hughes, P. A., Harrington, A. M., Castro, J., Liebregts, T., Adam, B., Grasby, D. J., Isaacs, N. J., Maldeniya, L., Martin, C. M., Persson, J., Andrews, J. M., Holtmann, G., Ashley Blackshaw, L., \& Brierley, S. M. (2013). Sensory neuro-immune interactions differ between Irritable Bowel Syndrome subtypes. Gut, 62(10), 1456-1465. https://doi.org/10.1136/gutjnl-2011-301856

Ibeakanma, C., \& Vanner, S. (2010). TNFa is a key mediator of the pronociceptive effects of mucosal supernatant from human ulcerative colitis on colonic DRG neurons. Gut, 59(5), 612-621. https://doi.org/10.1136/gut.2009.190439 
Jin, X., \& Gereau IV, R. W. (2006). Acute p38-mediated modulation of tetrodotoxin-resistant sodium channels in mouse sensory neurons by tumor necrosis factor-a. Journal of Neuroscience, 26(1), 246-255. https://doi.org/10.1523/JNEUROSCI.3858-05.2006

Kamada, N., Hisamatsu, T., Okamoto, S., Chinen, H., Kobayashi, T., Sato, T., Sakuraba, A., Kitazume, M. T., Sugita, A., Koganei, K., Akagawa, K. S., \& Hibi, T. (2008). Unique CD14+ intestinal macrophages contribute to the pathogenesis of Crohn disease via IL23/IFN-y axis. Journal of Clinical Investigation, 118(6), 2269-2280.

https://doi.org/10.1172/JCl34610

Khan, A. A., Diogenes, A., Jeske, N. A., Henry, M. A., Akopian, A., \& Hargreaves, K. M. (2008). Tumor necrosis factor a enhances the sensitivity of rat trigeminal neurons to capsaicin. Neuroscience, 155(2), 503-509.

https://doi.org/10.1016/j.neuroscience.2008.05.036

Lin, R. L., Gu, Q., \& Lee, L. Y. (2017). Hypersensitivity of vagal pulmonary afferents induced by tumor necrosis factor alpha in mice. Frontiers in Physiology, 8, 411.

https://doi.org/10.3389/fphys.2017.00411

McMahon, S. B., La Russa, F., \& Bennett, D. L. H. (2015). Crosstalk between the nociceptive and immune systems in host defence and disease. Nature Reviews Neuroscience, 16(7), 389-402. https://doi.org/10.1038/nrn3946

Ness, T. J., \& Gebhart, G. F. (1988a). Characterization of neurons responsive to noxious colorectal distension in the T13-L2 spinal cord of the rat. Journal of Neurophysiology, 60(4), 1419-1438. https://doi.org/10.1152/jn.1988.60.4.1419

Ness, T. J., \& Gebhart, G. F. (1988b). Colorectal distension as a noxious visceral stimulus: physiologic and pharmacologic characterization of pseudaffective reflexes in the rat. Brain Research, 450(1-2), 153-169. https://doi.org/10.1016/0006-8993(88)91555-7

Prado, J., Westerink, R. H. S., Popov-Celeketic, J., Steen-Louws, C., Pandit, A., Versteeg, S., van de Worp, W., Kanters, D. H. A. J., Reedquist, K. A., Koenderman, L., Hack, C. E., Eijkelkamp, N. (2021). Cytokine receptor clustering in sensory neurons with an engineered cytokine fusion protein triggers unique pain resolution pathways, 118(11), 2009647118. https://doi.org/10.1073/pnas.2009647118/-/DCSupplemental

Rijnierse, A., Koster, A. S., Nijkamp, F. P., \& Kraneveld, A. D. (2006). TNF-a is crucial for the development of mast cell-dependent colitis in mice. American Journal of Physiology - Gastrointestinal and Liver Physiology, 291(5), 969-976. https://doi.org/10.1152/ajpgi.00146.2006

Spiller, R., \& Major, G. (2016). IBS and IBD-separate entities or on a spectrum? Nature Reviews Gastroenterology and Hepatology, 13(10), 613-621.

https://doi.org/10.1038/nrgastro.2016.141

Thakur, M., Crow, M., Richards, N., Davey, G. I. J., Levine, E., Kelleher, J. H., Agley, C. C., Denk, F., Harridge, S. D. R., \& McMahon, S. B. (2014). Defining the nociceptor transcriptome. Frontiers in Molecular Neuroscience, 7, 87. https://doi.org/10.3389/FNMOL.2014.00087/ABSTRACT

van Wanrooij, S. J. M., Wouters, M. M., van Oudenhove, L., Vanbrabant, W., Mondelaers, S., Kollmann, P., Kreutz, F., Schemann, M., \& Boeckxstaens, G. E. (2014). Sensitivity Testing in Irritable Bowel Syndrome With Rectal Capsaicin Stimulations: Role of TRPV1 
Upregulation and Sensitization in Visceral Hypersensitivity? American Journal of Gastroenterology, 109(1), 99-109. https://doi.org/10.1038/ajg.2013.371

Wangzhou, A., Mcllvried, L. A., Paige, C., Barragan-Iglesias, P., Shiers, S., Ahmad, A., Guzman, C. A., Dussor, G., Ray, P. R., Gereau, R. W. 4th, \& Price, T. J. (2020). Pharmacological target-focused transcriptomic analysis of native vs cultured human and mouse dorsal root ganglia. Pain, 161(7), 1497-1517. https://doi.org/10.1097/J.PAIN.0000000000001866

Zeisel, A., Hochgerner, H., Lönnerberg, P., Johnsson, A., Memic, F., van der Zwan, J., Häring, M., Braun, E., Borm, L. E., La Manno, G., Codeluppi, S., Furlan, A., Lee, K., Skene, N., Harris, K. D., Hjerling-Leffler, J., Arenas, E., Ernfors, P., Marklund, U., \& Linnarsson, S. (2018). Molecular Architecture of the Mouse Nervous System. Cell, 174(4), 999-1014.e22. https://doi.org/10.1016/J.CELL.2018.06.021 